PROCEEDINGS OF THE AMERICAN MATHEMATICAL SOCIETY

Volume 124, Number 6, June 1996

\title{
REPRESENTATIONS AT FIXED POINTS OF SMOOTH ACTIONS OF COMPACT CONNECTED LIE GROUPS
}

\author{
HUAJIAN YANG
}

(Communicated by Thomas Goodwillie)

\begin{abstract}
Let $G$ be a compact connected Lie group acting smoothly on a connected closed manifold $M$ with nonempty fixed point set $F$. In this paper, we study the relation between the cohomology of $M$ or $M_{G}$ and the equivalent representations of $G$ at fixed points.
\end{abstract}

\section{INTRODUCTION}

Throughout this paper, we assume that $Q$ is the rational field and $G$ a compact connected Lie group acting smoothly on a connected closed manifold $M$ with fixed point set $F$. Let $M_{G}$ be the Borel construction associated with the $G$ action on $M$. Let $T(M)$ denote the tangent bundle of $M$ and $T_{x}(M)$ the tangent space at $x \in M$. For each $x \in F$, the induced $G$ linear action on the tangent space $T_{x}(M)$ of $M$ at $x \in F$ defines a real representation of $G$, which is denoted by $\Theta_{x}$. Let $R O(G)$ and $R U(G)$ be the real and complex representation rings of $G$ respectively. There is a complexification map $R O(G) \rightarrow R U(G)$, which is injective for a compact connected Lie group $G$. Denote the complexification of $\Theta_{x}$ also by $\Theta_{x}$. Recall that $M$ is totally nonhomologous to zero in $M_{G}$ with coefficient in $Q$ if the fibre inclusion $j: M \rightarrow M_{G}$ induces a surjection in cohomology $H^{*}(-; Q)([5$, p. 373]).

In this paper, we prove

Theorem 1.1. Let $G$ be a compact connected Lie group acting smoothly on a connected closed manifold $M$ with nonempty fixed point set $F$. Then $\Theta_{x}=\Theta_{y}$ for any $x, y \in F$, if one of the following conditions is satisfied:

(i) $\widetilde{K}(M) \otimes Q$ is trivial, or

(ii) $M$ is totally nonhomologous to zero in $M_{G}$ with coefficient in $Q$, and $H^{*}(M ; Q)$ is algebraically generated by some elements $\left\{x_{i}\right\}$ of odd degrees.

Note that the Chern character ch: $K(M) \otimes Q \rightarrow \bigoplus_{i>0} H^{2 i}(M ; Q)$ is an isomorphism ([7]). Thus condition (i) in the above theorem is equivalent to the condition that $H^{2 i}(M ; Z)$ is finite for all $0<2 i \leq \operatorname{dim}(M)$.

Now let $T=\left(S^{1}\right)^{r}$ be a fixed maximal torus of a compact connected Lie group $G$. It is known that two representations of $G$ are equivalent iff their restrictions on $T$ are equivalent ([6, Corollary 1.8.3]). Thus we reduce the problem of equivalent representations of $G$ to the case when $G$ is a torus. It is well known that

$$
R U\left(\left(S^{1}\right)^{r}\right)=Z\left\{t_{1}, t_{2}, \ldots, t_{r}\right\},
$$

Received by the editors September 22, 1993 and, in revised form, September 16, 1994.

1991 Mathematics Subject Classification. Primary 57S15. 
the finite Laurent series ring in $t_{i}$, where $t_{i}$ is the 1-dimensional complex representation of the $i$ th copy $S^{1}$ of $\left(S^{1}\right)^{r}$, given by

$$
t_{i}(z)(w)=z w, \quad z \in S^{1}, w \in C .
$$

Let $I\left(\left(S^{1}\right)^{r}\right)$ be the ideal of $R U\left(\left(S^{1}\right)^{r}\right)$ generated by $1-t_{1}, 1-t_{2}, \ldots, 1-t_{r}$. In [4, Theorem VI], Bredon proved

Theorem. Suppose the compact connected Lie group G acts smoothly on a connected manifold $M$ with nonempty fixed point set $F$. Assume that

$$
\pi_{2 i}(M) \text { is finite for all }\left\{\begin{array}{l}
1 \leq i \leq k-1 \quad \text { for general } G, \\
2 \leq i \leq k-1 \quad \text { for semi-simple } G .
\end{array}\right.
$$

Then $\Theta_{x}-\Theta_{y}$ is in the ideal $(I(T))^{k}$ of $R U(T)$ for any fixed points $x, y$.

Note that the manifold $M$ in Bredon's theorem is not necessarily closed. But we require $M$ to be closed in our theorems, since we will use the fact that the $K$-theory is representable only in the category of finite CW-complexes, that is, $\widetilde{K}(X) \approx \widetilde{K}^{0}(X)$ if $X$ is a finite CW-complex, where $\widetilde{K}^{*}(-)$ is the reduced cohomology represented by the well-known spectrum $K([9$, pp. 216,210$])$. By using the cohomology $H^{*}(M ; Z)$, we will prove the following

Theorem 1.2. Let $G$ be a compact connected Lie group acting smoothly on a connected closed manifold $M$ with nonempty fixed point set $F$. Then $\Theta_{x}-\Theta_{y} \in(I(T))^{n}$, if

$$
H^{2 i}(M ; Z) \text { is finite for all } 1 \leq i \leq n-1 .
$$

Moreover, if $T(M) \otimes C$ is stably trivial in $K\left(M^{(2 n)}\right) \otimes Q$, then $\Theta_{x}-\Theta_{y}$ is in $(I(T))^{n+1}$, where $M^{(2 n)}$, which contains at least one fixed point, is the (2n)-skeleton of a $G$-CW-structure of $M$.

Note that, as a CW-complex, the $(2 n)-G$-CW-skeleton $M^{(2 n)}$ might have cells of dimensions $>2 n$, since $G$ is connected. Actually by [8], the $G$-space $M^{(k)} / M^{(k-1)}$ is a wedge of based $G$-spheres

$$
G / H \times S^{k} /(G / H \times *)
$$

which is $(k-1)$-connected. Here $H$ is some closed isotropy subgroup of $G$.

As a specific example of applications of these theorems, we prove

Corollary 1.3. Suppose $G$ acts smoothly on a connected closed manifold $M$ with nonempty fixed point set $F$. Suppose $M$ is a rational homology sphere of dimension $n$. If $n$ is odd, then $\Theta_{x}=\Theta_{y}$ for $x, y \in F$. If $n$ is even, then there are at most two different representations $\Theta_{x}, x \in F$, up to equivalency.

\section{Proofs of the theorems}

Recall, if $X$ is a $G$-space, then the equivariant complex $K$-theory $K_{G}(X)$ is formed from the free abelian group on the equivalence classes of $G$-complex vector bundles over $X$ modulo the subgroup generated by $[\xi \oplus \eta]-[\xi]-[\eta]$. Its ring structure is induced by the tensor product of $G$-complex vector bundles. For a single point $*, K_{G}(*)$ is just the representation ring $R U(G)$.

Let $p_{0}: E_{G} \rightarrow B_{G}$ be the universal principal $G$-bundle. Let $B_{G}^{(r)}$ be the $r$ skeleton of $B_{G}$, and $E_{G}^{(r)}$ the inverse image $p_{0}^{-1}\left(B_{G}^{(r)}\right)$. For $G=S^{1}, E_{G}$ can be taken 
to be the infinite sphere $S^{\infty}=\bigcup S^{2 m+1}$, and $B_{G}$ the infinite complex projective space $C P^{\infty}$. Therefore we have $B_{G}^{(2 k)}=C P(k)=B_{G}^{(2 k+1)}$, and $E_{G}^{(2 k)}=E_{G}^{(2 k+1)}=$ $S^{2 k+1}$, when $G=S^{1}$. Note that any $G$ vector bundle over $E_{G}$ (resp. $\left.E_{G}^{(r)}\right)$ induces a vector bundle over $B_{G}$ (resp. $\left.B_{G}^{(r)}\right)$. By [1, Proposition 1.6.1], this gives an isomorphism $K_{G}\left(E_{G}\right) \rightarrow K\left(B_{G}\right)\left(\operatorname{resp} . K_{G}\left(E_{G}^{(r)}\right) \rightarrow K\left(B_{G}^{(r)}\right)\right)$. Let

$$
\alpha^{(r)}: R U(G) \rightarrow K_{G}\left(E_{G}^{(r)}\right)\left(\approx K\left(B_{G}^{(r)}\right)\right)
$$

be the homomorphism induced by the projection $E_{G}^{(r)} \rightarrow *$. By [1, Corollary 2.7.6, p. 105], if $G=S^{1}$, then the sequence

$$
0 \rightarrow R U(G) \stackrel{\varphi}{\longrightarrow} R U(G) \stackrel{\alpha^{(2 n-1)}}{\longrightarrow} K_{G}\left(E_{G}^{(2 n-1)}\right) \rightarrow 0
$$

is exact. Here the injectivity of $\varphi$ follows from the fact that $\varphi$ is the multiplication by $(1-t)^{n}$ when $G=S^{1}$ ([5, p. 357]).

Let $G$ act smoothly on $M$. Define the $G$ action on $E_{G} \times M$ or $E_{G}^{(2 m+1)} \times M$ to be the diagonal action. Then $M_{G}=\left(E_{G} \times M\right) / G$. Let $R^{m}(G)=\left(E_{G}^{(2 m+1)} \times M\right) / G$. Note that the $G$ action on $M$ induces a $G$ structure on the tangent bundle $T(M)$, and the projection

$$
E_{G} \times M \rightarrow M \quad\left(\text { or } E_{G}^{(2 m+1)} \times M \rightarrow M\right)
$$

is $G$-equivariant. Then the $G$ vector bundle $T(M)$ induces a $G$ vector bundle over $E_{G} \times M\left(\right.$ or $\left.E_{G}^{(2 m+1)} \times M\right)$, pulling back by the above projection, thus defines a vector bundle $\bar{T}(M)$ over $M_{G}$ (or a vector bundle $\bar{T}_{m}(M)$ over $R^{m}(G)$ ), which is called the tangent bundle along the fibres of the related fibre bundle $([2])$. Obviously, $i^{*}(\bar{T}(M))=\bar{T}_{m}(M)$, where $i: R^{m}(G) \rightarrow M_{G}$ is the inclusion. Also for $x \in F$, there exists a section $\rho_{x}$ for the projection $p: R^{m}(M) \rightarrow C P(m)$. The point is, if we regard $\alpha^{(2 n-1)}\left(\Theta_{x}\right)$ as an element of $K\left(B_{G}^{(2 n-1)}\right)\left(\approx K_{G}\left(E_{G}^{(2 n-1)}\right)\right)$, then

$$
\alpha^{(2 n-1)}\left(\Theta_{x}\right)=\rho_{x}^{*}\left(\bar{T}_{m}(M) \otimes C\right),
$$

where $\rho_{x}^{*}\left(\bar{T}_{m}(M) \otimes C\right)$ is the bundle induced by $\rho_{x}$. The bundle $\bar{T}_{m}(M) \otimes C$ will provide us a global view for the local complex representations $\Theta_{x}$. The following theorem is similar to $[4$, Theorem $\mathrm{V}]$.

Theorem 2.1. Let $G=S^{1}$ act smoothly on a connected closed manifold $M$ with nonempty fixed point set $F$. If $H^{2 i}(M ; Z)$ is finite for all $1 \leq i \leq n-1$, then

$$
\alpha^{(2 n-1)}\left(\Theta_{x}-\Theta_{y}\right)=0
$$

in $K(C P(n-1))$, and $\Theta_{x}-\Theta_{y}$ is divisible by $(1-t)^{n}$. Moreover, if $T(M) \otimes C$ is stably trivial over $K\left(M^{(2 n)}\right) \otimes Q$, then $\Theta_{x}-\Theta_{y}$ is divisible by $(1-t)^{n+1}$. Here $M^{(2 n)}$, which contains at least one fixed point, is the $(2 n)$-skeleton of a $G$-CW-structure of $M$.

Let $K$ and $H$ be the ring spectra corresponding to the nonconnective complex $K$-theory and the ordinary integral homology respectively. For a spectrum $E$, let $E_{(Q)}$ be the localization of $E$ at the rational field $Q$ in the Bousfield sense ([3]). Then $E_{(Q)}$ is a spectrum with $\pi_{k}\left(E_{(Q)}\right) \approx \pi_{k}(E) \otimes Q$. In particular, for the ring spectrum $H$,

$$
H_{(Q)}^{*}(Y) \approx H^{*}(Y ; Q) \approx H^{*}(Y ; Z) \otimes Q
$$

where $Y$ is a $\mathrm{CW}$-complex. In general, we have 
Lemma 2.2. Let $E$ be a spectrum and $Y$ a finite $C W$-complex. Then

$$
E_{(Q)}^{*}(Y) \approx E^{*}(Y) \otimes Q .
$$

Proof. Let $Y^{(n)}$ be the $n$-skeleton of $Y$. Since the cohomology represented by $E$ satisfies the wedge axiom $([9, \mathrm{p} .146])$ and the functor $\otimes Q$ commutes with finite products of abelian groups, the lemma is true if $Y$ is a finite wedge of spheres $\left\{S_{\alpha}^{m}\right\}$ of the same dimension $m$. Note that both $E_{Q}^{*}(Y)$ and $E^{*}(Y) \otimes Q$ are vector spaces over $Q$. This means we can do the induction from lower-dimensional skeletons of $Y$ to the higher skeletons, by using the exact sequences associated with $E_{Q}^{*}(-)$ and $E^{*}(-) \otimes Q$ for the pair $\left(Y^{(n)}, Y^{(n-1)}\right)$. Then the lemma follows.

Proof of Theorem 2.1. The proof here is similar to that of [11, Theorem 1.1]. Consider the Leray-Serre spectral sequences $\left\{E_{r}^{p, q}(i) ; d_{r}^{(i)}\right\}$ with local coefficients (which are actually constant) given by $H_{(Q)}^{*}(M)$ and $H_{(Q)}^{*}(\mathrm{pt}), i=1,2$, converging to $H_{(Q)}^{*}\left(R^{k}(M)\right)$ and $H_{(Q)}^{*}(C P(k))$ respectively $([9$, p. 350] or $[10$, p. 630]), with

$$
\begin{aligned}
& E_{2}^{p, q}(1)=H^{p}\left(C P(k) ; H_{(Q)}^{q}(M)\right), \\
& E_{2}^{p, q}(2)=H^{p}\left(C P(k) ; H_{(Q)}^{q}(\mathrm{pt})\right) .
\end{aligned}
$$

Also consider the morphism

$$
p^{*}: E_{r}^{p, q}(2) \rightarrow E_{r}^{p, q}(1)
$$

of related spectral sequences induced by the projection $p: R^{k}(M) \rightarrow C P(k)$. Since

$$
H_{(Q)}^{i}(M)=0 \quad \text { if } i \text { is even and } 2 \leq i \leq 2 n-2,
$$

we see at stage 2 that the morphism $p^{*}$ is an isomorphism if $p+q$ is even and $0 \leq p+q \leq 2 n-1$. Now the spectral sequence $E_{r}^{p, q}(2)$ collapses and all nontrivial elements on stage 2 survive to infinity. Thus the images of $p^{*}$ are all permanent cocycles. Since the projection $p$ has a section $\rho_{x}$, the nontrivial images of $p^{*}$ also survive to infinity when $0 \leq p+q \leq 2 n-2$. Therefore the morphism $p^{*}: E_{r}^{p, q}(2) \rightarrow$ $E_{r}^{p, q}(1)$ is an isomorphism for all $r \geq 2$ if $p+q$ is even and $0 \leq p+q \leq 2 n-1$, which induces an isomorphism

$$
p^{*}: H_{(Q)}^{i}(C P(k)) \rightarrow H_{(Q)}^{i}\left(R^{k}(M)\right)
$$

for $i$ even and $0 \leq i \leq 2 n-1$.

Next we consider the Atiyah-Hirzebruch-Whitehead spectral sequences $\left\{E_{r}^{p, q}(i)\right.$, $\left.d_{r}^{(i)}\right\}([9$, p. 340] or $[10$, p. 630]), $i=3,4$, built up from the CW-skeleton filtrations of $R^{k}(M)$ and $C P(k)$, and converging to $K_{(Q)}^{*}\left(R^{k}(M)\right)$ and $K_{(Q)}^{*}(C P(k))$ respectively, with

$$
\begin{aligned}
& E_{2}^{p, q}(3)=H^{p}\left(R^{k}(M) ; K_{(Q)}^{q}(\mathrm{pt})\right)=H_{(Q)}^{p}\left(R^{k}(M) ; K^{q}(\mathrm{pt})\right), \\
& E_{2}^{p, q}(4)=H^{p}\left(C P(k) ; K_{(Q)}^{q}(\mathrm{pt})\right)=H_{(Q)}^{p}\left(C P(k) ; K^{q}(\mathrm{pt})\right) .
\end{aligned}
$$

Let

$$
p^{*}: E_{r}^{p, q}(4) \rightarrow E_{r}^{p, q}(3)
$$

be the morphism of related spectral sequences induced by the projection $p$. Then, at stage $2, p^{*}$ is an isomorphism if $p$ is even and $0 \leq p \leq 2 n-2$. Since the spectral sequence $\left\{E_{r}^{p, q}(4), d_{r}^{(4)}\right\}$ collapses and the projection $p$ has a section $\rho_{x}$, we see that 
$p^{*}: E_{r}^{p, q}(4) \rightarrow E_{r}^{p, q}(3)$ is an isomorphism for $r \geq 2$ if $p$ is even and $0 \leq p \leq 2 n-2$. Thus

$$
p^{*}: K_{(Q)}^{0}(C P(k)) \rightarrow K_{(Q)}^{0}\left(R^{k}(M)\right)
$$

is an isomorphism up to the elements of filtrations $>2 n-1$, that is,

$$
p^{*}: K_{(Q)}^{0}(C P(k)) / F_{2 n} \rightarrow K_{(Q)}^{0}\left(R^{k}(M)\right) / G_{2 n}
$$

is an isomorphism, where $F_{2 n}, G_{2 n}$ are subgroups of elements of filtrations $>2 n-$ 1 of related groups. Let $\eta: K \rightarrow K_{(Q)}$ be the Bousfield localization and $\eta^{*}$ : $K^{*}(X) \rightarrow K_{(Q)}^{*}(X)$ the induced homomorphism. Note that $K^{0}(X) \approx K(X)$ if $X$ is a finite CW-complex. Thus we may regard $\eta^{*}$ to be defined on $K(X)$. Choose $k>2 n+1$ and assume in $K_{(Q)}^{0}\left(R^{k}(M)\right)$

$$
\eta^{*}\left(\bar{T}_{k}(M) \otimes C\right)=p^{*}(\xi)+a,
$$

where $a \in K_{(Q)}^{0}\left(R^{k}(M)\right)$ is an element of filtration $>(2 n-1)$, and $\xi \in K_{(Q)}^{0}(C P(k))$.

Let $j: B_{G}^{(2 n-1)} \rightarrow C P(k)$ be the inclusion. Consider the homomorphism $\alpha^{(2 n-1)}: R U\left(S^{1}\right) \rightarrow K\left(B_{G}^{(2 n-1)}\right)$. Since by $(2), \alpha^{(2 n-1)}\left(\Theta_{x}\right)=\rho_{x}^{*}\left(\bar{T}_{k}(M) \otimes C\right)$, we have

$$
\begin{aligned}
\eta^{*} \alpha^{(2 n-1)}\left(\Theta_{x}\right) & =\eta^{*} j^{*} \rho_{x}^{*}\left(\bar{T}_{k}(M) \otimes C\right)=j^{*} \rho_{x}^{*} \eta^{*}\left(\bar{T}_{k}(M) \otimes C\right) \\
& =j^{*} \rho_{x}^{*}\left(p^{*}(\xi)+a\right)=j^{*}(\xi),
\end{aligned}
$$

where the last equality is due to the fact that the element $a$ is of filtration $>(2 n-1)$, thus $j^{*} \rho_{x}^{*}(a)=0$. Consequently, $\eta^{*} \alpha^{(2 n-1)}\left(\Theta_{x}\right)$ is independent of the choices of $x \in F$, and $\eta^{*} \alpha^{(2 n-1)}\left(\Theta_{x}-\Theta_{y}\right)=0$ for any $x, y \in F$.

Note that $B_{G}^{(2 n-1)}$ is $C P(n-1)$, since $G=S^{1}$. By Lemma 2.2 and the structure of $K^{0}(C P(n-1))$, we see that $\eta^{*}: K^{0}(C P(n-1)) \rightarrow K_{(Q)}^{0}(C P(n-1))$ is injective. Thus $\alpha^{(2 n-1)}\left(\Theta_{x}-\Theta_{y}\right)=0$ for any $x, y \in F$. Therefore

$$
\Theta_{x}-\Theta_{y} \in \operatorname{ker}\left(\alpha^{(2 n-1)}\right)=I\left(S^{1}\right)^{n},
$$

which implies that $\Theta_{x}-\Theta_{y}$ is divisible by $(1-t)^{n}$. This completes the proof for the first statement.

We now consider the last statement. First, we have the exact sequence

$$
\widetilde{K}_{(Q)}^{0}\left(M^{(2 n)}\right) \stackrel{f^{*}}{\longleftarrow} \widetilde{K}_{(Q)}^{0}\left(R^{k}\left(M^{(2 n)}\right)\right) \stackrel{g^{*}}{\longleftarrow} K_{(Q)}^{0}\left(R^{k}\left(M^{(2 n)}\right), M^{(2 n)}\right),
$$

where $f: M^{(2 n)} \rightarrow R^{k}\left(M^{(2 n)}\right)$ and $g: R^{k}\left(M^{(2 n)}\right) \rightarrow\left(R^{k}\left(M^{(2 n)}\right), M^{(2 n)}\right)$ are the inclusion and the projection respectively. Let $\lambda-m$ be the class in $\widetilde{K}_{(Q)}^{0}\left(R^{k}\left(M^{(2 n)}\right)\right)$ which corresponds to $i^{*} \eta^{*}\left(\bar{T}_{k}(M) \otimes C\right)$, where $i: R^{k}\left(M^{(2 n)}\right) \rightarrow R^{k}(M)$ is the inclusion and $m$ is the complex dimension of $\bar{T}_{k}(M) \otimes C$. Then $f^{*}(\lambda-m)$ is zero by the assumed condition. Thus by the exactness,

$$
\lambda-m=g^{*}(\zeta)
$$

for some $\zeta \in K_{(Q)}^{0}\left(R^{k}\left(M^{(2 n)}\right), M^{(2 n)}\right)$.

Similar to what we did for the first statement, we consider the Leray-Serre spectral sequences $\left\{E_{r}^{p, q}(i) ; d_{r}^{(i)}\right\}$ with coefficients given by $H_{(Q)}^{*}\left(M^{(2 n)}\right)$ and $H_{(Q)}^{*}(\mathrm{pt})$, 
converging to $H_{(Q)}^{*}\left(R^{k}\left(M^{2 n}\right), M^{(2 n)}\right)$ and $\widetilde{H}_{(Q)}^{*}(C P(k))$ for $i=5,6$ respectively, with

$$
\begin{gathered}
E_{2}^{p, q}(5)=\widetilde{H}^{p}\left(C P(k) ; H_{(Q)}^{q}\left(M^{(2 n)}\right)\right), \\
E_{2}^{p, q}(6)=\widetilde{H}^{p}\left(C P(k) ; H_{(Q)}^{q}(\mathrm{pt})\right) .
\end{gathered}
$$

Let $\left(p_{1}^{\prime}\right)^{*}: E_{r}^{p, q}(6) \rightarrow E_{r}^{p, q}(5)$ be the morphism of related spectral sequences induced by $p_{1}^{\prime}$, where $p_{1}^{\prime}:\left(R^{k}\left(M^{(2 n)}\right), M^{(2 n)}\right) \rightarrow(C P(k), *)$ is the projection induced by the bundle projection $p_{1}: R^{k}\left(M^{(2 n)}\right) \rightarrow C P(k)$. Since $M^{(2 n)}$ contains at least one fixed point $x, p_{1}^{\prime}$ has a section $\rho_{x}$. By the fact that

$$
H_{(Q)}^{i}\left(M^{(2 n)}\right)=0 \quad \text { if } i \text { is even and } 2 \leq i \leq 2 n-2,
$$

at stage 2 , we see $\left(p_{1}^{\prime}\right)^{*}$ is an isomorphism if $p+q$ is even and $0 \leq p+q \leq 2 n$. Now the spectral sequence $E_{r}^{p, q}(6)$ collapses and all nontrivial elements on stage 2 survive to infinity. Thus the images of $\left(p_{1}^{\prime}\right)^{*}$ are permanent cocycles, and the nontrivial images of $\left(p_{1}^{\prime}\right)^{*}$ survive to infinity, for $p_{1}^{\prime}$ has a section $\rho_{x}$. This implies that $\left(p_{1}^{\prime}\right)^{*}: E_{r}^{p, q}(6) \rightarrow E_{r}^{p, q}(5)$ is an isomorphism for $p+q$ even and $0 \leq p+q \leq 2 n$, and $r \geq 2$. Thus

$$
\left(p_{1}^{\prime}\right)^{*}: \widetilde{H}_{(Q)}^{i}(C P(k)) \rightarrow H_{(Q)}^{i}\left(R^{k}\left(M^{(2 n)}\right), M^{(2 n)}\right)
$$

are isomorphisms if $i$ is even and $0 \leq i \leq 2 n$.

Next consider the Atiyah-Hirzebruch-Whitehead spectral sequences

$$
\left\{E_{r}^{p, q}(i), d_{r}^{(i)}\right\}, \quad i=7,8,
$$

built up by the CW-skeleton filtrations of $\left(R^{k}\left(M^{(2 n)}\right), M^{(2 n)}\right)$ and $(C P(k), *)$, and converging to $K_{(Q)}^{*}\left(R^{k}\left(M^{(2 n)}\right), M^{(2 n)}\right)$ and $\widetilde{K}_{(Q)}^{*}(C P(k))$ respectively, with

$$
\begin{gathered}
E_{2}^{p, q}(7)=H^{p}\left(R^{k}\left(M^{(2 n)}\right), M^{(2 n)} ; K_{(Q)}^{q}(\mathrm{pt})\right) \\
=H_{(Q)}^{p}\left(R^{k}\left(N^{(2 n)}\right), M^{(2 n)} ; K^{q}(\mathrm{pt})\right), \\
E_{2}^{p, q}(8)=\widetilde{H}^{p}\left(C P(k) ; K_{(Q)}^{q}(\mathrm{pt})\right)=\widetilde{H}_{(Q)}^{p}\left(C P(k) ; K^{q}(\mathrm{pt})\right) .
\end{gathered}
$$

Note that at stage $2,\left(p_{1}^{\prime}\right)^{*}: E_{2}^{p, q}(8) \rightarrow E_{2}^{p, q}(7)$ is an isomorphism if $p$ is even and $0 \leq p \leq 2 n$. Similar to what we did in the first statement for the spectral sequences $\left\{E_{r}^{p, q}(i) ; d_{r}^{(i)}\right\}$ with $i=3,4$, we see

$$
\left(p_{1}^{\prime}\right)^{*}: \widetilde{K}_{(Q)}^{0}(C P(k)) \rightarrow K_{(Q)}^{0}\left(R^{k}\left(M^{(2 n)}\right), M^{(2 n)}\right)
$$

is an isomorphism up to filtrations $>2 n$. Therefore we may assume $\zeta=\left(p_{1}^{\prime}\right)^{*}(c)+$ $a$ in $K_{(Q)}^{0}\left(R^{k}\left(M^{(2 n)}\right), M^{(2 n)}\right)$, where $c \in \widetilde{K}_{(Q)}^{0}(C P(k))$, and the element $a$ is of filtration $>2 n$. Thus in $\widetilde{K}_{(Q)}^{0}\left(R^{k}\left(M^{(2 n)}\right)\right)$

$$
i^{*} \eta^{*}\left(\bar{T}_{k}(M) \otimes C\right)-m=g^{*}\left(p_{1}^{\prime}\right)^{*}(c)+g^{*}(a) .
$$

Let $h_{x}: B_{G}^{(2 n)} \rightarrow R^{k}\left(M^{(2 n)}\right)$ be the CW-approximation of the composition

$$
B_{G}^{(2 n)} \stackrel{j}{\rightarrow} C P(k) \stackrel{\rho_{x}}{\longrightarrow} R^{k}(M) .
$$


Then

$$
\begin{aligned}
\eta^{*} \alpha^{(2 n)}\left(\Theta_{x}\right) & =\eta^{*} j^{*} \rho_{x}^{*}\left(\bar{T}_{k}(M) \otimes C\right)=j^{*} \rho_{x}^{*} \eta^{*}\left(\bar{T}_{k}(M) \otimes C\right) \\
& =h_{x}^{*} i^{*} \eta^{*}\left(\bar{T}_{k}(M) \otimes C\right)=h_{x}^{*} g^{*}\left(p_{1}^{\prime}\right)^{*}(c)+h_{x}^{*} g^{*}(a)+m \\
& =h_{x}^{*} g^{*}\left(p_{1}^{\prime}\right)^{*}(c)+m=h_{x}^{*} p_{1}^{*} j_{0}^{*}(c)+m=j^{*} j_{0}^{*}(c)+m
\end{aligned}
$$

from the commutative diagram

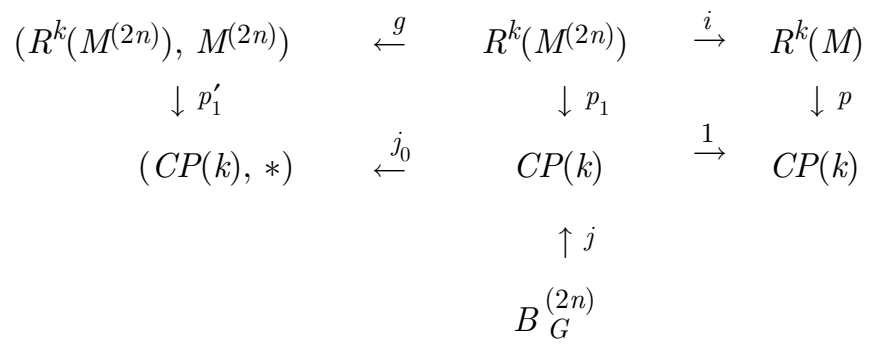

where $j_{0}$ is the ordinary projection $C P(k) \rightarrow(C P(k), *)$. Here the fifth equality is due to the fact that the element $a$ is of filtration $>2 n$. The sixth equality is from the fact $p_{1}^{\prime} g=j_{0} p_{1}$. The last equality follows from the fact that $i h_{x}$ is homotopic to $\rho_{x} j$, thus $p i h_{x}\left(=p_{1} h_{x}\right)$ is homotopic to $p \rho_{x} j(=j)$. This shows $\eta^{*} \alpha^{(2 n)}\left(\Theta_{x}\right)$ is independent of the choices of $x \in F$ and $\eta^{*} \alpha^{(2 n)}\left(\Theta_{x}-\Theta_{y}\right)=0$ for any $x, y \in F$. Since $B_{G}^{(2 n)}=C P(n)$ and $\eta^{*}: K^{*}(C P(n)) \rightarrow K_{(Q)}^{*}(C P(n))$ is injective, we have $\alpha^{(2 n)}\left(\Theta_{x}-\Theta_{y}\right)=0$. The last statement follows from the fact that $\operatorname{ker}\left(\alpha^{(2 n)}\right)=\operatorname{ker}\left(\alpha^{(2 n+1)}\right)$.

Proof of Theorem 1.2. The proof is similar to that of [4, Theorem VI]. By considering a fixed maximal torus $T$ of $G$, we may reduce $G$ to the case when $G=\left(S^{1}\right)^{r}$. Consider the map $S^{1} \rightarrow\left(S^{1}\right)^{r}$ given by $z \rightarrow\left(z^{n_{1}}, z^{n_{2}}, \ldots, z^{n_{r}}\right)$, which induces a homomorphism $R U\left(\left(S^{1}\right)^{r}\right) \rightarrow R U\left(S^{1}\right)$ given by $t_{i} \rightarrow t^{n_{i}}$, where $n_{1}, n_{2}, \ldots, n_{r}$ are integers. Suppose

$$
\Theta_{x}-\Theta_{y}=P\left(t_{1}, t_{2}, \ldots, t_{r}\right) \in R U\left(\left(S^{1}\right)^{r}\right) .
$$

Then, by Theorem 2.1, $P\left(t^{n_{1}}, t^{n_{2}}, \ldots, t^{n_{r}}\right)$ is divisible by $(1-t)^{n}$ (or $(1-t)^{n+1}$ when $T(M) \otimes C$ is stably trivial in $K\left(M^{(2 n)}\right) \otimes Q$ ) for any integers $n_{1}, n_{2}, \ldots, n_{r}$. An argument on elementary algebra, as claimed in [4], shows this is equivalent to $P\left(t_{1}, t_{2}, \ldots, t_{r}\right) \in\left(I\left(\left(S^{1}\right)^{r}\right)\right)^{n}\left(\operatorname{resp} .\left(I\left(\left(S^{1}\right)^{r}\right)\right)^{n+1}\right)$.

Proof of Theorem 1.1. Note that in condition (ii), $M$ is totally nonhomologous to zero in $M_{G}$ with coefficient in $Q$ implies that $M$ is totally nonhomologous to zero in $M_{S^{1}}$ with coefficient in $Q$ for any circle subgroup of $G$. Then similar to the proof of Theorem 1.2, we may assume $G=S^{1}$ for both cases (i) and (ii). By the exact sequence (1), it suffices to prove $\alpha^{(2 k+1)}\left(\Theta_{x}-\Theta_{y}\right)=0$ for all $k>0$ and $x, y \in F$.

For (i), we consider the Leray-Serre spectral sequences $\left\{E_{r}^{p, q}(i), d_{r}^{(i)}\right\}, i=9,10$, with

$$
\begin{aligned}
E_{2}^{p, q}(9) & =H^{p}\left(C P(k), K_{(Q)}^{q}(M)\right), \\
E_{2}^{p, q}(10) & =H^{p}\left(C P(k), K_{(Q)}^{q}(\mathrm{pt})\right),
\end{aligned}
$$


converging to $K_{(Q)}^{0}\left(R^{k}(M)\right)$ and $K_{(Q)}^{0}(C P(k))$ respectively. Note that

$$
E_{2}^{p, q}(9)=H^{p}\left(C P(k), K_{(Q)}^{q}(M)\right)=H^{p}\left(C P(k), K^{q}(M) \otimes Q\right),
$$

and the morphism $p^{*}: E_{r}^{p, q}(10) \rightarrow E_{r}^{p, q}(9)$ is an isomorphism at $r=2$ if $p+q$ is even. With a similar argument as for the spectral sequences $\left\{E_{r}^{p, q}(i), d_{r}^{(i)}\right\}, i=3,4$, in the proof of Theorem 2.1, we see that

$$
p^{*}: K_{(Q)}^{0}(C P(k)) \rightarrow K_{(Q)}^{0}\left(R^{k}(M)\right)
$$

is an isomorphism. Thus we may assume

$$
\eta^{*}\left(\bar{T}_{k}(M) \otimes C\right)=p^{*}(\xi),
$$

where $\xi \in K_{(Q)}^{0}(C P(k))$. Then, similar to the proof of Theorem 2.1,

$$
\eta^{*} \alpha^{(2 k+1)}\left(\Theta_{x}\right)=\rho_{x}^{*} \eta^{*}\left(\bar{T}_{k}(M) \otimes C\right)=\rho_{x}^{*} p^{*}(\xi)=\xi \in K_{(Q)}^{0}(C P(k)),
$$

which is independent of the choices of $x \in F$. Therefore $\alpha^{(2 k+1)}\left(\Theta_{x}-\Theta_{y}\right)=0$ for any $x, y \in F$. Thus $\Theta_{x}=\Theta_{y}$ by (1).

Consider statement (ii). Since $M$ is totally nonhomologous to zero in $M_{G}$ with coefficient in $Q$ implies that $M$ is totally nonhomologous to zero in $R^{k}(M)$ with coefficient in $Q$ for any $k \geq 0$, we see that $H^{*}\left(R^{k}(M) ; Q\right)$ is generated by some $\left\{1, c_{i}\right\}$ and some products of two or more $c_{i}$ as a module over $H^{*}(C P(k) ; Q)$ for any $k>0$, where $c_{i}$ is of odd degree. Consider the homomorphism $\rho_{x}^{*}: H^{*}\left(R^{k}(M) ; Q\right) \rightarrow$ $H^{*}(C P(k) ; Q)$. Then we have $\rho_{x}^{*}\left(c_{i}\right)=0$, since the degree of $c_{i}$ is odd. Thus $\rho_{x}^{*}$ is independent of the choices of $x \in F$.

Now let $X$ be a finite CW-complex and

$$
\text { ch : } K_{(Q)}^{0}(X)=K^{0}(X) \otimes Q \rightarrow H^{* *}(X ; Q)
$$

the Chern character, where $H^{* *}(X)=\bigoplus_{i=0}^{\infty} H^{2 i}(X ; Q)$. Then ch is an isomorphism $([7])$ and we have the following commutative diagram:

$$
\begin{array}{ccc}
K_{(Q)}^{0}\left(R^{k}(M)\right) & \stackrel{c h}{\rightarrow} & \left.H^{* *}\left(R^{k}(M)\right) ; Q\right) \\
\downarrow \rho_{x}^{*} & & \downarrow \rho_{x}^{*} \\
\left.K_{(Q)}^{0}\left(C P^{k}\right)\right) & \stackrel{c h}{\rightarrow} & H^{* *}(C P(k) ; Q)
\end{array}
$$

Since $\rho_{x}^{*}: H^{* *}\left(R^{k}(M) ; Q\right) \rightarrow H^{* *}(C P(k) ; Q)$ is independent of the choices of $x \in F$, the map $\rho_{x}^{*}: K_{(Q)}^{0}\left(R^{k}(M)\right) \rightarrow K_{(Q)}^{0}(C P(k))$ is independent of the choices of $x \in F$ by diagram (3). Thus $\rho_{x}^{*}\left(\bar{T}_{k}(M) \otimes C\right) \in K^{0}(C P(k))$ is independent of the choices of $x \in F$ by the commutative diagram

$$
\begin{array}{ccc}
K^{0}\left(R^{k}(M)\right) & \stackrel{\eta^{*}}{\rightarrow} & K_{(Q)}^{0}\left(R^{k}(M)\right) \\
\downarrow \rho_{x}^{*} & & \downarrow \rho_{x}^{*} \\
K^{0}(C P(k)) & \stackrel{\eta^{*}}{\rightarrow} & K_{(Q)}^{0}(C P(k))
\end{array}
$$

where the $\eta^{*}$ in the bottom row is injective, and the proof for (ii) follows. 
Proof of Corollary 1.3. If $n$ is odd, then, by using the Atiyah-Hirzebruch-Whitehead spectral sequence with $E_{2}^{p, q}=\widetilde{H}^{p}\left(M ; K_{(Q)}^{q}(\mathrm{pt})\right)$ converging to $\widetilde{K}_{(Q)}^{*}(M)$, we have $\widetilde{K}_{(Q)}^{0}(M)=0$. This means $\widetilde{K}^{0}(M) \otimes Q=0$ by Lemma 2.2 , and $\Theta_{x}=\Theta_{y}$ by Theorem 1.1(i).

Now let $n$ be even. Similar to the proof of Theorem 1.2, we may assume $G=S^{1}$. Consider the Leray-Serre spectral sequence $\left\{E_{r}^{p, q}, d_{r}\right\}$ with $E_{2}^{p, q}=$ $H^{p}\left(C P^{\infty} ; H_{(Q)}^{q}(M)\right)$, converging to $H_{(Q)}^{*}\left(M_{G}\right)$. Obviously, this spectral sequence collapses. Thus $H_{(Q)}^{*}\left(M_{G}\right)$ is a free $H_{(Q)}^{*}\left(C P^{\infty}\right)$ module with a basis $\{1, c\}$. Since we are working on the coefficient $Q$, we may require $c^{2} \in p^{*} H_{(Q)}^{*}\left(C P^{\infty}\right)$. Actually, if

$$
c^{2}=p^{*}(a) c+p^{*}\left(b^{2}\right),
$$

then we can replace $c$ by $c^{\prime}=c-\frac{1}{2} p^{*}(a)$ and see $\left(c^{\prime}\right)^{2} \in p^{*} H_{(Q)}^{*}\left(C P^{\infty}\right)$. Let $\rho_{x}^{*}(c)=b_{x}$. Then $c^{2}$ is in the image of $p^{*}$ implies $\left(b_{x}\right)^{2}=\left(b_{y}\right)^{2}$ for $x, y \in F$. Thus $\rho_{x}^{*}(c)=\rho_{y}^{*}(c)$ or $-\rho_{y}^{*}(c)$.

Now the Leray-Serre spectral sequence associated with $R^{k}(M)$ collapses, and $H_{(Q)}^{*}\left(R^{k}(M)\right)$ is a free $H_{(Q)}^{*}(C P(k))$ module with a basis $\left\{1, c^{\prime}\right\}$. By the map of Leray-Serre spectral sequences induced by the inclusion $j: R^{k}(M) \rightarrow M_{G}$, we may require $c^{\prime}=j^{*}(c)$. Thus by diagrams (3) and (4) again, if $\rho_{x}^{*}(c)=\rho_{y}^{*}(c)$ in $H^{*}\left(C P^{\infty} ; Q\right)$, then $\alpha^{(2 k+1)}\left(\Theta_{x}-\Theta_{y}\right)=0$ for any $k \geq 0$. This means $\Theta_{x}=\Theta_{y}$. Since we have at most two different morphisms

$$
\rho_{x}^{*}: H^{*}\left(M_{G} ; Q\right) \rightarrow H^{*}\left(C P^{\infty} ; Q\right),
$$

there are at most two representations $\Theta_{x}$ for $x \in F$ up to equivalency.

\section{ACKNOWLEDGMENTS}

I would like to thank the referee for his valuable comments, in particular, his pointing out to me that the $n$ in Corollary 1.3 must be odd in order to get $\Theta_{x}=\Theta_{y}$. Special thanks to Professors D. M. Davis, Zhende Wu, Zaisi Zuo, and Zhongze Liu, from whom I learned algebraic topology. I am also grateful to Lehigh University for the financial support while this work was performed.

\section{REFERENCES}

1. M. F. Atiyah K-theory, Benjamin, New York, 1967. MR 36:7130

2. A. Borel and Hirzebruch, On the characteristic classes of the homogeneous spaces, Amer. J. Math. 80 (1958), 458-538. MR 21:1586

3. A. K. Bousfield, The localization of spectra with respect to homology, Topology 18 (1979), 257-281. MR 80m:55006

4. G. E. Bredon, Representations at fixed points of smooth actions of compact groups, Ann. of Math. (2) 89 (1969), 512-532. MR 39:7628

5. _ Introduction to compact transformation groups, Academic Press, New York and London, 1972. MR 54:1265

6. Wu Yi Hsiang, Cohomology theory of topological transformation groups, Springer-Verlag, New York, Heidelberg, and Berlin, 1975. MR 54:11363

7. Max Karoubi, K-theory, An introduction, Springer-Verlag, Berlin, Heidelberg, and New York, 1978. MR 58:7605

8. J. P. May, Equivariant homotopy and homology theory, Contemp. Math., vol. 12, Amer. Math. Soc., Providence, RI, 1982, 209-217. MR 83m:55011

9. R. M. Swizter, Algebraic topology — homotopy and homology, Springer-Verlag, New York, Heidelberg, and Berlin, 1975. MR 52:6695 
10. G. W. Whitehead, Elements of homotopy theory, Springer-Verlag, New York, Heidelberg, and Berlin, 1978. MR 80b:55001

11. Huajian Yang, Representations at fixed points of smooth actions of finite groups, (to appear). CMP 95:03

Department of Mathematics, Lehigh University, Bethlehem, Pennsylvania 18015

E-mail address: hyo2@lehigh.edu

Department of Mathematics, South China Normal University, Guangzhou 510631, People's Republic of China 